\title{
aulaabierta
}

www.elsevier.es/aulaabierta

\section{Calidad de vida en niños pequeños portadores de un implante coclear}

\author{
Ana Carolina Camacho Castro ${ }^{\mathrm{a}}$, Antonio Hernández Fernández ${ }^{\mathrm{b},{ }^{*}}$ e Isabel María Ferrándiz Vindel ${ }^{\mathrm{c}}$ \\ a Departamento de Pedagogía, Universidad de Jaén, España \\ ${ }^{\mathrm{b}}$ Departamento de Pedagogía, Universidad de Jaén, España \\ cUniversidad de Castilla-La Mancha, España
}

INFORMACIÓN DEL ARTÍCULO

\section{Historia del artículo:}

Recibido el 30 de noviembre de 2011

Aceptado el 7 de octubre de 2013

\section{Palabras clave:}

Implante coclear

Educación inclusiva

Comunicación oral

Logopedia

Calidad de vida

Keywords:

Cochlear implant

Inclusive education

Oral communication

Speech

Quality of life

\section{R E S U M E N}

En este artículo se presenta una investigación desarrollada durante un curso escolar en la Comunidad Autónoma de Andalucía (2010-2011). El tema central del estudio es analizar la influencia del implante coclear en la mejora de la calidad de vida de niños que lo han recibido en sus primeros años de vida. No es el propósito de este trabajo describir íntegramente la investigación, sino que de forma selectiva se hace un recorrido por el diseño metodológico y los resultados de la investigación, que nos lleva a plantear una serie de reflexiones finales acerca de la mejora de la forma de vida y del desarrollo personal de niños que padecen hipoacusia y son portadores de un implante coclear.

(C) 2011 Instituto de Ciencias de la Educación de la Universidad de Oviedo. Publicado por Elsevier España, S.L. Todos los derechos reservados.

\section{Quality of life in children with a cochlear implant}

\section{A B S T R A C T}

This article describes a study conducted in an Andalusia Community school (year 2010-2011). It studied the influence of cochlear implantation on improving the quality of life of the children who received implants during their early years. The methodological design and results of the study are also presented, in order to find a better solution to improve the life conditions and personal development of children suffering hearing loss, and having a cochlear implant.

(C) 2011 Instituto de Ciencias de la Educación de la Universidad de Oviedo. Published by Elsevier España, S.L. All rights reserved.

\section{Introducción}

La calidad de vida relacionada con la salud (CVRS) es un concepto multidimensional que se basa en la percepción subjetiva del individuo. Las dimensiones principales de las medidas de CVRS son las funciones física, mental y social. La medición de la CVRS se refiere a todos los aspectos que experimenta directamente el individuo, como las funciones física, mental y social y la percepción general de la salud (Badía, 2005).

Entender el problema de la pérdida de calidad de vida y el incremento de ésta proporcionado por el implante coclear resulta

\footnotetext{
* Autor para correspondencia: Antonio Hernández Fernández. Departamento de Pedagogía, Universidad de Jaén. Campus Las Lagunillas, CP 23071, Jaén (España).

Correo electrónico: ahernand@ujaen.es
}

bastante complejo en el caso de la hipoacusia. La hipoacusia, por definición, supone una pérdida de la capacidad auditiva y una limitación sensorial en sî; supone una reducción en la calidad de vida. Sin embargo, las consecuencias de la hipoacusia van generalmente más allá, y dependen del grado de pérdida auditiva y la forma en que ésta se instaura en relación con la edad o el momento de adquisición del lenguaje. Uno de los principales factores que condiciona la pérdida de calidad de vida asociada a la hipoacusia es la consecuencia que tiene sobre la comunicación oral. La comunicación es una de las dimensiones más importantes para la realización del individuo como ser humano, y la comunicación oral es el medio más común de interacción entre la mayoría de las personas (Redondo, 1959).

En el caso de los niños, a la dificultad comunicativa se suma la dificultad en el desarrollo de las habilidades lingüísticas, ya que el aprendizaje del lenguaje se produce en los primeros años de vida. Un diagnóstico tardío del problema provocará importantes restricciones en el acceso al lenguaje oral y, por tanto, limitaciones en 
el lenguaje comunicativo dentro del entorno social. Esto incidirá de forma negativa en los ámbitos educativo, social, familiar, personal y laboral del niño. De aquí nuestro propósito de centrar este trabajo en niños pequeños.

Las variables que condicionan la evolución de un niño con implante coclear son múltiples (Manrique y Morera, 2006) y deben ser consideradas a la hora de pensar en la candidatura de niño para implante, durante el período de evaluación y para el tratamiento específico que tendrá una vez implantado. Es necesario considerar variables relacionadas con los profesionales, variables intrínsecas del niño que recibirá el implante y variables relacionadas con su familia.

Después de la colocación del implante coclear, viene el período de intervención logopédica. Esta intervención es diferente en función de una serie de factores. Los factores más determinantes para diseñar un programa de intervención son la edad de aparición de la hipoacusia y el tiempo transcurrido entre la aparición de ésta y el momento de la implantación (Camacho, Colmenero y Hernández, 2005).

No se puede describir un programa universal para todos los niños. Cada programa debe ser particular y específico, tomando la información necesaria para que el niño alcance niveles de percepción y procesamiento cada vez más complejos (Furmanski, 2003).

La duración del programa de intervención depende de factores como la edad, la motivación o el nivel de lenguaje previo a la implantación, pero el más determinante es el tiempo transcurrido entre la aparición de la hipoacusia y la implantación. Cuanto menor es este intervalo de tiempo, más rápida es la evolución. Si el implante se realiza durante la primera infancia, el programa postimplante se inscribe dentro del programa educativo, que abarca no sólo la audición, el lenguaje y el habla, sino el desarrollo cognitivo, afectivo y social del niño (Manrique, 2005).

La edad de recepción del implante coclear es el factor que más fuertemente condiciona la mejora de la calidad de vida de los niños implantados. Cuanto menor es la edad de implantación, mayores son los resultados, a largo plazo, aunque también es mayor el tiempo necesario para alcanzarlos (Sainz, Vega, Padilla y Camacho, 2005, p. 28).

Hay que tener en cuenta la diversidad de los factores pronóstico que afectan a los resultados obtenidos con el implante coclear, para prever de forma realista los beneficios que se esperan en cada caso. Esta información ayudará a fijar objetivos educativos, logopédicos y psicológicos, y a planificar adecuadamente el proceso de rehabilitación o habilitación posterior a la colocación del implante.

Un aspecto que dificulta el estudio de los factores pronóstico y de los resultados es el elevado número de variables involucradas en el proceso de implantación coclear, así como el alto grado de multidisciplinariedad en la actuación profesional. En los resultados obtenidos van a influir aspectos educativos (modalidad de escolarización o de rehabilitación logopédica), aspectos relacionados con la implicación familiar (modalidad de comunicación utilizada, asistencia a actividades formativas), aspectos médicos (anatómicos, fisiológicos) y aspectos relacionados con el propio niño (motivación respecto al proceso de rehabilitación, situación social) (Sainz et al., 2005).

El objetivo general de este estudio es analizar la influencia del implante coclear en la mejora de la calidad de vida de niños que han recibido un implante coclear entre los 0 y 3 años de edad. Para ello, se han tenido en cuenta una serie de variables, de diferente naturaleza. Algunas de estas variables están presentes antes de la implantación (variables preimplante), y otras lo están después (variables postimplante). Al mismo tiempo, se ha considerado una serie de indicadores de calidad de vida, siguiendo las investigaciones relacionadas con el tema (Manrique y Morera, 2006).

\section{Método}

\section{Participantes}

La muestra de este estudio son niños que han recibido el implante en el Hospital Universitario San Cecilio de Granada, hospital de referencia en Andalucía (España) para el tratamiento de hipoacusias, y que ya en octubre de 2007 contaba con un total de 544 pacientes portadores de un implante coclear, procedentes en su mayoría de todas las provincias de Andalucía.

Del total de pacientes-niños de este centro hospitalario, se han seleccionado 97 siguiendo una técnica de muestreo intencional o deliberado. Los criterios que se han considerado como requisitos necesarios para pertenecer a la muestra han sido que padeciesen una hipoacusia neurosensorial profunda bilateral de tipo prelocutivo, que hubiesen recibido su implante coclear entre los 0 y 3 años, que contasen con un período de evolución postimplante de entre 1,5 y 5 años, y que no presenten otras patologías asociadas a la hipoacusia.

\section{Instrumento de evaluación}

En esta investigación, el instrumento de medida utilizado ha sido diseñado para este caso, con el fin de lograr la información necesaria para ir dando respuesta al objeto problema de estudio que nos planteamos. Consiste en un cuestionario ad-hoc elaborado a partir de dos factores que pueden condicionar la evolución de estos niños (modo de comunicación utilizado por los padres pre- y postimplante, y tipo de escolarización pre- y postimplante) y de cuatro indicadores de calidad (preguntar por la procedencia de los sonidos, utilizar el lenguaje oral de forma espontánea, utilizar un lenguaje inteligible para desconocidos, y seguir instrucciones verbales).

Se trata de una escala de tipo Likert, en la que se ha pedido que califiquen los enunciados presentados del 0 al 5 según su grado de acuerdo con cada uno de ellos. Estas afirmaciones pueden reflejar actitudes positivas hacia algo o negativas. Las primeras se denominan favorables y las segundas desfavorables.

La entrega para la realización de los cuestionarios se ha llevado a cabo de manera directa, tratando de que hubiese representantes de todos las personas que están en contacto de un modo u otro con los niños que han recibido el implante coclear. Por un lado, se empezó entregando el cuestionario a miembros del equipo sanitario del hospital. Seguidamente, se pasó a todos los padres de la muestra seleccionada. El cuestionario es el mismo para todos ellos. No obstante, cada uno presenta una numeración y codificación diferente para diferenciar la información, entre equipo sanitario y familia, preservando eso sí, el anonimato de todos ellos.

\section{Análisis de datos}

El objetivo fundamental de este trabajo fue comparar los resultados obtenidos antes y después del implante tras un período de evolución de entre 1,5 y 5 años. La hipótesis general base de este estudio es que existen diferencias significativas entre la situación pre- y postimplante en niños hipoacúsicos. En concreto, se quiso analizar si existen o no diferencias en los dos factores y los cuatro indicadores de calidad del cuestionario. Para poder contrastar esta hipótesis, se empleó el estadístico $t$ de Student para muestras relacionadas, para la comparación de medias pretest-postest.

\section{Resultados}

En la tabla 1 se presentan los resultados del contraste de medias en las seis variables analizadas. Los datos obtenidos indican que no existen diferencias entre la situación pre- y postimplante en el modo 
Tabla 1

Comparación de medias pretest-postest en las seis variables evaluadas $(\mathrm{n}=97)$

\begin{tabular}{|c|c|c|c|c|c|}
\hline & \multicolumn{2}{|c|}{ Pretest } & \multicolumn{2}{|c|}{ Postest } & \multirow[t]{2}{*}{ Sig. } \\
\hline & M & DT & M & DT & \\
\hline Modo de comunicación & 1,21 & 0,59 & 1,33 & 0,75 & - \\
\hline Tipo de escolarización & 3,52 & 0,89 & 1,93 & 0,48 & * \\
\hline Preguntar por la procedencia y origen de los sonidos & 1,08 & 0,37 & 2,08 & 1,22 & * \\
\hline Utilizar el lenguaje oral de forma espontánea & 1,03 & 0,23 & 4,16 & 0,95 & * \\
\hline Utilizar un lenguaje inteligible para desconocidos & 1,00 & 0,00 & 3,59 & 0,99 & * \\
\hline Seguir instrucciones & 1,00 & 0,00 & 3,95 & 0,99 & $*$ \\
\hline
\end{tabular}

${ }^{*} \mathrm{p} \leq 0,001$.

DT: desviación típica; M: media.

de comunicación utilizado por los padres $(t=-1,486 ; p=0,140)$. Sí existen diferencias entre la situación pre- y postimplante en el tipo de escolarización $(t=16,016 ; p=0,000)$.

Respecto a los indicadores de calidad de vida, existen diferencias estadísticamente significativas pretest-postest en preguntar por el origen y procedencia de los sonidos $(t=-15,074 ; p=0,000)$, uso espontáneo del lenguaje oral ( $t=-32,376 ; p=0,000)$, usar un lenguaje inteligible $(t=-25,823 ; p=0,000)$, y seguir instrucciones $(t=-29,231 ; p=0,000)$.

\section{Discusión y conclusiones}

Tras el análisis de los resultados obtenidos, podemos afirmar que existen diferencias entre la situación pre- y postimplante respecto al tipo de escolarización, preguntar por la procedencia del sonido, usar el lenguaje oral de forma espontánea, usar un lenguaje inteligible para desconocidos, y seguir instrucciones verbales.

Podemos añadir también que, para un primer análisis sobre la pérdida de calidad de vida e incremento de la misma proporcionado por un implante coclear, es posible utilizar un instrumento elaborado ad-hoc cercano a los profesionales que trabajan con estos casos y sobre todo a las familias de los usuarios.

Los resultados obtenidos apuntan asimismo a la dificultad que tienen los niños con hipoacusia neurosensorial profunda bilateral de tipo prelocutivo en el desarrollo de las habilidades lingüísticas. Ello apoyaría la necesidad de una evaluación logopédica y de una intervención específica lo más pronto posible. Se han podido identificar algunos indicadores que pueden favorecer la "habilitación auditiva".

Hemos constatado también, a lo largo de nuestro trabajo, que es conveniente conocer en profundidad algunos indicadores de CVRS antes de diseñar y ejecutar un método de rehabilitación logopédica en niños con un implante coclear. Por ello se ha adoptado una metodología cuantitativa de análisis para identificar, interpretar y describir lo que está ocurriendo con algunos indicadores en niños que han recibido su implante coclear entre los 0 y los 3 años.
Las diferencias significativas entre la situación preimplante y postimplante, respecto a los indicadores de calidad de vida, son direccionales: en todos los indicadores en los que las diferencias han sido estadísticamente significativas se ha observado un aumento en la frecuencia. Aumenta la frecuencia con la que los niños preguntan por el origen y procedencia de los sonidos, la frecuencia con la que usan el lenguaje oral de forma espontánea, la frecuencia con la que su lenguaje es inteligible para desconocidos, y la frecuencia con la que son capaces de seguir instrucciones verbales.

No se han hallado cambios en el tipo de comunicación utilizado por los padres en la situación pre- y postimplante. Podemos añadir algunas observaciones que se nos han hecho sobre la falta de información precisa, antes de la obtención del diagnóstico médico; el sentir que están logrando objetivos específicos, por pequeños que sean y que están progresando; el contacto con familias de niños en condiciones similares para ayudar a que las expectativas sean adecuadas y realistas, etc.

Se han observado diferencias respecto al tipo de escolarización, ya que en la situación preimplante, la mayoría de la muestra no tenía la edad suficiente para estar escolarizada.

\section{Referencias bibliográficas}

Badía, X. (2005). Estudios de calidad de vida. Cuadernos de atención primaria. Barcelona: Ars Medica.

Camacho, A. C., Colmenero, M. J., y Hernández, A. (2005). Entrenamiento auditivo en niños portadores de un implante coclear. Departamento de Pedagogía, Universidad de Jaén. Recuperado el 13 de marzo de 2011 de http://doces.es/uploads/articulos/ ntrenamiento-auditivo-en-niNos-portadores-de-un-implante-coclear.pdf

Furmanski, H. (2003). Implantes cocleares en niños: (Re) habilitación auditiva y terapia auditiva verbal. Barcelona: Ediciones Nexus.

Manrique, M. (2005). Implantes cocleares. Barcelona: Masson.

Manrique, M., y Morera, C. (2006). Evaluación del implante coclear como técnica de tratamiento de la hipoacusia profunda en pacientes pre y postlocutivos. Acta Otorrinolaringología Española, 57, 2-23.

Redondo, E. (1959). Educación y comunicación. Madrid: C.S.I.C.

Sainz, M., Vega, A., Padilla, J. L., y Camacho, A. C. (2005). Evaluación de la efectividad terapéutica del tratamiento de hipoacusias severas y profundas en niños: implante coclear versus audífono. Costes, beneficios y mejora en la calidad de vida. Madrid: Ministerio de Sanidad y Consumo. 